\title{
An investigation of the relationship between eye and retinal image movement in the perception of movement ${ }^{1}$
}

ARIEN MACK ${ }^{2}$ NEW SCHOOL FOR SOCIAL RESEARCH

The question investigated was whether or not eye movements accompanied by abnormal retinal image movements, movements that are either or both at a different rate or in a different direction than the eye movement, predictably lead to perceived movement. Os reported whether or not they saw a visual target move when the movement of the target was either dependent on and simultaneous with their eye movements or when the target movement was independent of their eye movements. In the main experiment, observations were made when the ratio between eye and target movement $(\mathrm{em} / \mathrm{tm})$ was $2 / 5,1 / 5,1 / 10,1 / 20$, and 0 . All these ratios were tested when the direction of the target movement was in the same $\left(H^{+}\right)$, opposite $(\mathrm{H}-1)$, and at right angles to $\left(V+, V_{-}\right)$the movement of the eyes. Eye movements, target movements, and reports of target movement were recorded. Results indicate that a discrepancy between eye and target movement greater than 20\% predictably leads to perceived target movement, whereas a discrepancy of $5 \%$ or less rarely leads to perceived movement. The results are interpreted as support for the operation of a compensatory mechanism during eye movements.

What is the basis of perceiving the movement of a single object in an otherwise unstructured visual field? The most obvious answer is that it is the movement of the retinal image that is crucial, ${ }^{3}$ since with every movement of an object there is a corresponding movement of the image of that object across the retina of the $O$. This answer is the more attractive since there is now ample evidence that vertebrate nervous systems are well endowed with receptor cells uniquely sensitive to retinal movements. If, however, retinal displacement were the central factor in perceiving object movement, it would follow that every time there was retinal movement greater than the threshold of the detecting cell mechanisms, object movement- would be perceived, and in its absence, no such movement would be seen.

It is, of course, a well-known fact that the case is not so simple. There are too many instances of perceived movement in the absence of retinal movement and of the correlative phenomenon of no perceived movement despite the occurrence of retinal displacement to maintain that retinal image movement is either a sufficient or necessary condition for the perception of object movement. An afterimage viewed in the dark by an $O$ moving his eyes appears to move (Mack \& Bachant, 1969), but there is no retinal movement. A moving object in an otherwise empty field, accurately pursued by the $O$ 's eyes, appears to move, but there is no retinal movement. A stationary scene viewed by moving eyes appears stationary despite retinal image movements (position constancy). The existence of these and other phenomena suggests that the relationship between retinal image movement and perceived movement is more complex. A theory that attempts to specify the relationship between image or stimulus displacements and perceived movement has been variously referred to as a cancellation, discounting, compensating, or taking-into-account theory of movement perception (von Holst, 1954; Mackay, 1967; Rock, 1966; Teuber, 1960).

If we are permitted to overlook the subtle and not so subtle differences between the various statements of this theory, it becomes possible to set out its main outlines. All forms of this theory are in agreement that retinal image changes alone can neither account for the occurrence of movement perception nor alone determine whether the movement perceived resides in the object or in the $O$. It is a theory that accounts for perceived object motion as well as the faihure to perceive object motion despite displacements or changes in the retinal image. It assumes, in fact, that these are correlative events. The theory asserts that the basis of perceived object motion resides in the relationship between the behavior of the retinal image and the available sensory information about the movement of the $O$. It assumes the existence of some kind of central mechanism, a comparator (von Holst, 1954) that takes into account, matches, compares, or evaluates retinal image shifts with reference to information about $\mathbf{O}$ movement. The theory assumes that if not accounted for or matched by appropriate $O$ movements, retinal image displacements will invariably lead to the perception of object motion and correlatively if eye movements are not matched by retinal image displacements, movement will be perceived. Thus, when retinal image changes are "matched," constancy is predicted, and when those changes are not "matched," some change in visual perception is predicted. Constancy is predicted when retinal image changes are accounted for in terms of $\mathbf{O}$ movement information. Constancy here signifies the perception of stability in the visual field, i.e., objects in the visual field appear to maintain their positions despite displacements of the retinal images. Failures of constancy refer to those occasions in which image displacements of stationary objects lead to perceived movement in the visual field.

The postulation of some sort of comparator mechanism provides a concept that allows us to account for many of the facts of movement perception. There is now considerable evidence supporting the operation of this sort of mechanism during head movements. Information about head movements appears to mediate the relationship between visual displacement and perceived movement (Wallach \& Kravitz, 1965; Wallach \& Frey, 1969; Hay, 1968; Rock, 1966). In these studies, abnormal visual displacements, displacements that are either faster, slower, or in a different direction than the O's own head movements and do not simply reflect the $O$ movement, predictably led to perceived object motion. Until now there has been no available evidence that a comparator operates during eye movements. Since the eyes are rarely still and since we generally see our visual environment as stationary despite the constant displacements of the retinal images produced by the fairly constant movements of the eye, it would appear that some kind of comparator mechanism in fact operates during eye movements. ${ }^{4}$ Yet in the only completely reported experiment examining the relationship between eye movements, image movements, and the perception of movement (Wallach \& Lewis, 1965) the authors conclude that target movements are not assessed in terms of eye movements. By devising a situation in which the $O$ saw a projection of his own 
pupil, the authors produced abnormal displacements of a visual target dependent on eye movements. This technique made it possible to produce a situation in which the target disk, the projection of the pupil, could be made to move at either a slower or faster rate than the eye itself. Their results indicate that abnormal target displacements accompanying eye movements, do not reliably cause reports of perceived target movement. They conclude: "Our results are incompatible with the view that the apparent rest of visual objects whose images shift due to eye movements is to be explained by a compensating process which takes the eye movements into account."

From the Wallach and Lewis results it might be possible to argue that all detection of retinal displacement is suppressed during eye movements. If this were the case, then, since no retinal displacements would be registered during an eye movement, there would be no reason to expect a loss of position constancy during eye movements, and there would be no reason to postulate some kind of comparator that could match retinal displacement to $O$ movement information. That this cannot be the case may be seen from the fact that there is evidence that an afterimage appears to move even when it is viewed in complete darkness and the only $O$ movement is $O$ eye movement (Mack \& Bachant, 1969). This suggests that at least the absence of any retinal displacement during an eye movement constitutes a basis for movement perception. If the absence of displacement is crucial, then there must be a record of the occurrence of displacements in order that these two kinds of events be distinguished. It therefore appears that there must at least be a mechanism that records retinal displacements and eye movements and is capable of determining when these events occur simultaneously, which determination ordinarily results in no perceived object movement. If no evidence for such a mechanism is found to operate during eye movements, then it would seem to mean that no real (as opposed to illusory) movement could be perceived during an eye movement except when the image of the visual object is stable on the retina (as in the case of an accurately pursued target or afterimage).

To our knowledge the only available empirical evidence that supports the view that a comparator mechanism operates during eye movement appears in Yarbus (1967). In his book, Yarbus reports an experiment in which he produced abnormal image displacements by means of suction-contact lenses. Os report perceiving target motion. Unfortunately the account given is brief and no details are included.

The contradiction between the Wallach and Lewis results and those briefly reported by Yarbus made further examination of the relationship between eye movement, image movement, and perceived movement necessary. Another reason for such a study is the fact that an eye-movemen $t / i m a g e-m o v e m e n t$ comparator capable of doing nothing more than merely discriminating between instances of eye movements that are either accompanied or not accompanied by retinal displacements would make it very difficult to account for any perception of movement during eye movements.

By a technique different from that used by either Wallach and Lewis or Yarbus, a situation was created in which eye movements were accompanied by abnormal image displacements, that is, displacements that did not directly reflect the movements of the eye. Os reported whether or not the target at which they were looking appeared to move.

\section{METHOD}

The essential requirements for work of this kind are: a visual target whose movements are strictly related to the movements of the $O$ 's eyes, a means of altering the relation between eye and target movement, and a means of simultaneously recording eye movements and the reports of movement made by the $O$.

Lateral eye movements were recorded by means of electrooculography. Beckman bipotential skin electrodes were attached to the outer margins of the eyes and a third electrode was attached to the center of the $O$ 's forehead and served as a ground. The electrodes were wired through a noise filter to one channel of a Sanborn four-channel pen recorder with sufficient dc amplification to represent the signal from the eyes accurately. The output of this channel was wired in turn to an external volume control from which it was fed into either the horizontal or vertical input channel of either one or two Techtronix No. 502A oscilloscopes. The volume control permitted continuous control over variations in gain. The effect of this was to afford continuous control over the amount of oscilloscope trace movement produced by an eye movement. The oscilloscope trace served as the visual target. It was the only object visible to the $O$ during testing sessions. In order that the $\mathbf{O}$ should not see the glow of the oscilloscope screen itself, Kodak Wratten filters, No. 47B, were placed over the screen and during testing all Os wore goggles containing Kodak Wratten filter No. 35. The movements of the oscilloscope trace, which was approximately $2 \mathrm{~mm}$ in diam, were recorded by one channel of the pen recorder. Simultaneous recording of eye and target trace movement permitted a continuous check on the relation between eye and target movement. The volume control knob was calibrated from 0 to 1 . When this knob was set at 0 , the trace was not moved at all by eye movements. When this knob was set at 1 , the trace moved the same amount as the eyes and thus could approximate a stabilized image (i.e., when the direction of trace displacement and eye movement were the same). When the knob was set at some point between 1 and 0 , the trace moved some fraction of the amount the eyes moved. Thus, when the knob was set at $2 / 5$, the target trace moved $2 / 5$ of the distance that the eyes moved. (All calibrations were frequently checked.) The increase in what is best described as trace jitter with increase in gain were mostly eliminated by filtering. What jitter remained was accounted for in the experimental design.

A switch on the control panel permitted rapid shifts from the horizontal to the vertical input channels of the oscilloscopes. This switch had the effect of making the trace move either horizontally or vertically in response to lateral eye movements of the O. A second switch, a plus-minus switch, changed the direction of trace movement accompanying an eye movement. When this switch was set at + and the horizontal-vertical switch was set at horizontal, the trace moved in the same direction as the eyes. If the eyes moved right, the trace moved right. If the +switch was set at -, the trace moved left when the eye moved right. When the horizontal-vertical switch was set at vertical, then a + setting of the +- switch caused the trace to move up when the eyes moved right. A - setting caused the trace to move down when the eyes moved right. The various control switches permitted four directions of target movement, horizontal with the eyes $(\mathbf{H}+)$, horizontal against the eyes $(\mathrm{H}-)$, vertical and up with the eyes to the right $(\mathrm{V}+)$, and vertical down with eyes right ( $\mathrm{V}-$ ), as well as continuous control over the ratio of eye-to-target movement, $\mathrm{em} / \mathrm{tm}$. The following ratios of eye-to-target movement, $\mathrm{em} / \mathrm{tm}$, were repeatedly tested: $0,1 / 20$, $1 / 10,1 / 5,2 / 5$. Ratios of $1 / 2$ and 1 were tested only under conditions of random eye movements (to be explained).

Small instant on-off lamps were attached to both sides of one of the oscilloscope screens. Each light was a distance of $6.25 \mathrm{~cm}$ from the center of the screen. Switches on the control panel turned on either one or the other of these lights. Operations of one of these switches 
resulted in a light flash that lasted less than $1 / 20 \mathrm{sec}$. The lamps were coated and taped with an opaque material so that the light flashes were sufficiently dim that no afterimages were produced even in dark-adapted eyes. The light switches were wired to an event marker on the pen recorder providing a permanent record of the onset of light flashes.

Each oscilloscope was housed in a shielded and light-tight cell in which there was sufficient room for an $O$ to sit. The center of the oscilloscope screens coincided more or less with an eye level of the Os. Bite bars were fixed $27.5 \mathrm{~cm}$ in front of the oscilloscope screens and prevented head movements during testing. Positioned at an upright oblique angle and to the right of the screens were panels consisting of four buttons placed at the four points of a cross. These were used by the $O$ to report target movements. The top button was used to report upward movement, the bottom button for downward movement, the right button for rightward movement and the left button for leftward movement. The reporting buttons were wired through the dc amplifier to a channel on the pen recorder. Depression of one of these buttons moved the pen in that channel a specific amount, so that the distance the pen moved indicated which button was being pressed. The two oscilloscopes were run simultaneously and at all times displayed identical trace movement because the input to and the calibration of the oscilloscopes was always identical.

\section{Procedure}

The Os were run in pairs, one who observed a target whose behavior depended on his eye movements (experimental), and the other who observed the same target without its being dependent on his eye movement (control). Each $\mathrm{O}$ was tested twice, once as an experimental and once as a control $O$. The advantages of testing each $\mathrm{O}$ twice and of running $\mathrm{Os}$ in pairs are that it provides control data for each testing session, and it allows each $O$ to act as his own control, thus accounting for styles of reporting. Since we obtained data from two Os simultaneously, the occurrence of any electronic jitter with increases in gain was not significant, because we were able to analyze the difference between the reports of the two Os. The experimental $O$ wore the eye electrodes and sat in the oscilloscope cell containing the light-flashing apparatus. The control $O$ sat in the other oscilloscope cell, which was identical to that in which the experimental $O$ sat except that there were no flashing lights. There were two parts to each testing session. An entire testing session lasted about $3 / 4 \mathrm{~h}$. The two parts are designated directed eye movements (DEM) and nondirected eye movements (NDEM).

$D E M$. The experimental $O$ was instructed to look straight ahead at the oscilloscope trace, and bite on the bite bar. This signaled the onset of a trial. Immediately following instructions to look straight ahead and a check of the eye-movement record to establish that the O had properly responded, one of the light switches was flipped. The $O$ then turned his eyes to where he had seen the light flash and was required to keep his eyes in this position until he was once again instructed to look straight ahead. During the interval between turning his eyes to the flash and returning his eyes to straight ahead, $O$ reported if there had been any target trace movement. He reported whether or not the trace moved when he moved his eyes. All instructions were gone over carefully before testing occurred, and all testing was preceded by at least 10 practice trials in which all the $O$ was required to do was to move his eyes to the flashed light and hold them in that position until once again instructed to look straight ahead. Despite the apparent complexity of the O's task, most Os easily succeeded in doing what was required of them.

Control Os wore no eye electrodes and their eye movements were not recorded. They were asked to look straight ahead and bite on the bite bar on instruction from the $E$ (the same instructions were heard by both $O$ s) and to report if the trace moved within the interval between the straight-ahead instructions and the instructions to the experimental $\mathrm{O}$ to once again look straight ahead. Both Os were therefore reporting on the behavior of the trace during the eye movements of the experimental $\mathrm{O}$. Although no record of the eye movements of the control Os were made, it is probably safe to assume that control Os' reports were made on the basis of a period in which their eyes moved very little since they had been instructed to look straight ahead.

Each combination of displacement ratio and trace movement direction was presented in blocks of four trials separated by at least a brief rest period. Thus a block of trials consisted of four trials in which the $\mathrm{em} / \mathrm{tm}$ and the direction of target displacement was constant. Several of these blocks of trials were separated by longer rest intervals in which any dark adaptation was erased. Each block of four trials consisted of two left and two right light flashes presented in random order. In all testing sessions, each combination of target displacement direction and $\mathrm{em} / \mathrm{tm}$ ratio was presented for at least one block of trials. Both ascending and descending orders of $\mathrm{em} / \mathrm{tm}$ were used. Blocks of trials in which the $\mathrm{em} / \mathrm{tm}$ was zero were presented at least three times during each testing session. Zero testing trials were usually presented after a block of trials in which the em/tm was either $2 / 5$ or $1 / 20$. Blocks of trials were ordered so that four consecutive blocks of trials were presented in which the em/tm was constant while the direction of target displacement was varied from one block of trials to the next.

NDEM. This condition was included as an attempt to verify the difference between "incidental" and "voluntary" eye movements reported by Wallach and Lewis (1965). They found that abnormal image displacements accompanying eye movements sometimes led to movement reports when eye movements were voluntary but rarely when eye movements were incidental. In this condition both $O s$ received exactly the same instructions. They were directed to look continuously at the target trace and to indicate if it moved by pressing the right button of the button panel for as long as the trace continued to move. Unlike the DEM reports, only the presence or absence of movement and not its direction was reported. The task of constantly monitoring a small and dim target light was sufficiently taxing without requiring Os to also report direction of target movement.

The following $\mathrm{em} / \mathrm{tm}$ ratios were tested in combination with the four directions of trace movement: $1,1 / 2,2 / 5,1 / 5,1 / 10$, $1 / 20$, and 0 . Each combination of $\mathrm{em} / \mathrm{tm}$ ratio and direction of target displacement was presented for a 15 -sec interval at the end of which the $\mathrm{em} / \mathrm{tm}$ ratio, the direction of target displacement, or both were changed. Os were allowed to rest approximately every minute.

Half the Os began testing with DEM, while the remaining half began with NDEM. Half the Os were experimental Os before they were tested as controls. The remaining half was tested in the reverse order.

\section{Subjects}

Fourteen Ss (seven pairs) were each run twice. These Ss were Stanford University students who were paid for their participation.

\section{DEM}

\section{RESULTS}

Data from the DEM condition were analyzed in terms of the percent of correct responses in each block of four trials. For example, if a $S$ reported target movement to the left following eye movements to a right light flash, and target movement to the right following eye movements to a left light flash in three of the four trials constituting the block of trials in which the 
Table 1

Mean Percent Correct Movement Responses: DEM

\begin{tabular}{|c|c|c|c|c|c|c|c|c|c|c|}
\hline \multirow[b]{2}{*}{ EM/TM } & \multicolumn{2}{|c|}{$\mathbf{H}-$} & \multicolumn{2}{|c|}{$\mathrm{H+}$} & \multicolumn{2}{|c|}{$\mathbf{V +}$} & \multicolumn{2}{|c|}{$\mathbf{V}-$} & \multicolumn{2}{|c|}{ Grand $\bar{X}$} \\
\hline & $\mathbf{E}$ & C & $\mathbf{E}$ & C & $\mathbf{E}$ & $\mathrm{C}$ & $\mathbf{E}$ & $C$ & $\mathbf{E}$ & $\mathrm{C}$ \\
\hline $\begin{array}{l}2 / 5 \\
1 / 5 \\
1 / 10 \\
1 / 20 \\
0\end{array}$ & $\begin{array}{l}87.5 \\
78.5 \\
41.1 \\
13.2\end{array}$ & $\begin{array}{l}96.8 \\
91.1 \\
83.9 \\
64.6\end{array}$ & $\begin{array}{l}91.8 \\
89.6 \\
39.2 \\
11.4\end{array}$ & $\begin{array}{c}100 \\
94.6 \\
87.5 \\
64.3\end{array}$ & $\begin{array}{l}94.6 \\
87.5 \\
51.8 \\
12.5\end{array}$ & $\begin{array}{c}100 \\
100 \\
92.9 \\
75.7\end{array}$ & $\begin{array}{r}94.6 \\
87.5 \\
41.1 \\
7.8\end{array}$ & $\begin{array}{c}100 \\
98.2 \\
92.3 \\
76.9\end{array}$ & $\begin{array}{l}92.1 \\
85.7 \\
43.3 \\
11.2 \\
93.2\end{array}$ & $\begin{array}{l}99.2 \\
95.9 \\
89.1 \\
70.3 \\
81.7\end{array}$ \\
\hline
\end{tabular}

Table 2

Analysis of Variance Outcome (DEM)

\begin{tabular}{|c|c|c|c|}
\hline Factors & $\mathbf{F}$ & DF & \\
\hline $\begin{array}{l}\text { Groups } \\
\text { Experimental and Control }\end{array}$ & 177.546 & $1 / 416$ & $<.001$ \\
\hline $\begin{array}{l}\text { Direction } \\
\text { Horizontal and Vertical }\end{array}$ & 46.269 & $1 / 416$ & .05 \\
\hline $\begin{array}{l}\text { Direction } \\
\text { Positive and Negative }\end{array}$ & 1.044 & $1 / 416$ & \\
\hline $\begin{array}{l}\mathrm{EM} / \mathrm{TM} \\
2 / 5,1 / 5,1 / 10,1 / 20\end{array}$ & 121.147 & $3 / 416$ & $<.001$ \\
\hline
\end{tabular}

Table 3

Mean Scores and Critical Values for Estimating the Significance of Difference Between Means (Scheffe Procedure)

\begin{tabular}{|c|c|c|c|c|c|c|}
\hline \multirow[b]{2}{*}{ EM/TM } & \multicolumn{2}{|c|}{$\mathbf{H}$} & \multicolumn{2}{|c|}{$\mathbf{V}$} & \multicolumn{2}{|c|}{ Grand Mean } \\
\hline & $\mathbf{E}$ & C & $\mathbf{E}$ & C & $\mathbf{E}$ & $\mathrm{C}$ \\
\hline \multirow[t]{2}{*}{$\begin{array}{l}2 / 5 \\
1 / 5 \\
1 / 10 \\
1 / 20\end{array}$} & $\begin{array}{l}89.64 \\
84.11 \\
40.18 \\
10.18\end{array}$ & $\begin{array}{l}98.39 \\
92.86 \\
85.72 \\
76.25\end{array}$ & $\begin{array}{l}94.64 \\
87.68 \\
46.43 \\
12.33\end{array}$ & $\begin{array}{l}100 \\
99.11 \\
92.86 \\
64.46\end{array}$ & $\begin{array}{l}92.11 \\
85.89 \\
43.30 \\
11.25\end{array}$ & $\begin{array}{l}99.20 \\
95.98 \\
89.29 \\
70.36\end{array}$ \\
\hline & \multicolumn{4}{|c|}{$\begin{array}{l}\mathrm{CV}=48.83, P=.01 \\
\mathrm{CV}=40.49, \mathrm{P}=.05\end{array}$} & \multicolumn{2}{|c|}{$\begin{array}{l}C V=23.56, P=.01 \\
C V=20.72, P=.05\end{array}$} \\
\hline
\end{tabular}

$C V=$ Critical Value,$E=$ Experimental, $C=$ Control, $H=$ Horizontal, $V=$ Vertical

$\mathrm{em} / \mathrm{tm}$ level was $1 / 5$ and the direction of target movement was $\mathbf{H}-$, he received a score of $75 \%$. Table 1 presents the mean percent correct scores of all experimental and control Os for all combinations of target-movement direction and levels of $\mathrm{em} / \mathrm{tm}$. An analysis of variance was computed for this data. The results of this analysis appear in Table 2 . All three main factors had a significant effect on the perception of movement. Using the Scheffé procedure for all possible a posteriori comparisons (Winer, 1962) the differences between means obtained in each condition were evaluated. The outcome of this analysis is found in Table 3. Statistical comparisons between means obtained when target movement was horizontal and when it was vertical and when the $\mathrm{em} / \mathrm{tm}$ was constant yield no significance (see Table 3). These comparisons were made separately for experimental and control Os. The direction of target displacement does not appear to affect the perception of movement significantly under these conditions.

Again using the Scheffé procedure, all means obtained at one level of $\mathrm{em} / \mathrm{tm}$ were compared with the means obtained from the immediately adjacent $\mathrm{em} / \mathrm{tm}$ level. These comparisons were again made separately for experimental and control Os (see Table 3). For experimental Os, the mean percent correct responses for the $2 / 5$ $\mathrm{em} / \mathrm{tm}$ level is not significantly different from the mean percent correct responses for $\mathrm{em} / \mathrm{tm}$ equals $1 / 5$. Movement was reported as frequently when the target displaced $1 / 5$ the distance the eyes moved as when the target displaced $2 / 5$ of that distance. The following differences are significant: The difference between means in the $1 / 5 \mathrm{em} / \mathrm{tm}$ condition and means in the $1 / 10 \mathrm{em} / \mathrm{tm}$ condition and the difference between means in the $1 / 10$ and means in the $1 / 20 \mathrm{em} / \mathrm{tm}$ condition are all significant at greater than .01 . For all $\mathrm{em} / \mathrm{tm}$ levels less than $1 / 5$, the smaller the $\mathrm{em} / \mathrm{tm}$ ratio the more difficult it is for the $O$ to detect target displacement during an eye movement. This may be stated another way. The smaller the absolute extent of target displacement during an eye movement the less likely it is to be detected. The difference between means in conditions in which the $\mathrm{em} / \mathrm{tm}$ is $2 / 5$ and 0 are not significant. Ss appear to be as accurate in reporting no target movement during a saccade as target movement that is $2 / 5$ as great as the eye movement.

An analysis of means obtained in control conditions reveals the only significant difference to be that between the condition in which $\mathrm{em} / \mathrm{tm}$ was $1 / 20$ and all the remaining $\mathrm{em} / \mathrm{tm}$ conditions (see Table 3). Very small target displacements appear to be difficult to detect regardless of whether or not they are simultaneous with an eye movement. 5

Statistical comparisons were made in which each experimental $S$ was compared with himself as a control $O$ (see Table 3 ). The following differences were found to be significant: all differences between experimental and control $O s$ in conditions in which the em/tm equals $1 / 10$ (significant at greater than .01), and all differences between experimental and controls for $\mathrm{em} / \mathrm{tm}$ equals $1 / 20$ (significant at greater than .01). Thus, in the $0,2 / 5$, and $1 / 5$ conditions, there are no significant differences between experimental and control $\mathrm{Os}$ in their ability to perceive target movement, although the differences that do appear between these groups are all characterized by the fact that the control means are greater. When the $\mathrm{em} / \mathrm{tm}$ was $1 / 10$ or $1 / 20$ Os were able to more accurately detect target displacement in the control conditions than were those Os in the experimental conditions who were reporting on target displacements concurrent with eye movements.

An analysis of the errors made by experimental Os reveals no particular trend. This analysis is potentially most interesting in the case of errors made when the em/tm level is zero, since these errors might possibly reveal a tendency to see a stationary target moving in a direction predictably related to the direction of the eye movement. Errors were extremely few in this condition and reveal no trend. Most cases in which the percent correct scores were less than $100 \%$ were the result of a failure to report any movement rather than reports of movement in an objectively incorrect direction.

An analysis was made of reaction time (RT) to the light flashes in order to determine if on any trials the light was visible when the eyes began to move. Any trials in which this had occurred would have to have been ignored since reports of movement derived from these trials might have been based on the availability of object-relative displacement information. ${ }^{3}$ In no case did RT approximate the duration of the light flash, which was $1 / 20 \mathrm{sec}$. Typically, RT was slightly less than $1 / 2 \mathrm{sec}$. The only explanation we can offer for the prolonged duration of the RT is that the complexity of the O's task tended to make his responses to the light 
Table 4

Range (in Degrees) of Target Movement

\begin{tabular}{cc}
\hline $\mathrm{EM} / \mathrm{TM}$ & Range \\
\hline $2 / 5$ & $6.2-4.2$ \\
$1 / 5$ & $2.1-3.1$ \\
$1 / 10$ & $1.6-1.1$ \\
$1 / 20$ & $.8-.5$ \\
\hline
\end{tabular}

Table 5

Mean Percent Movement Reports: NDEM

\begin{tabular}{lll}
\hline & E & C \\
\hline 1 & 69.6 & 65.2 \\
$1 / 2$ & 55.0 & 59.8 \\
$2 / 5$ & 52.5 & 56 \\
$1 / 5$ & 35.0 & 40.3 \\
$1 / 10$ & 31.3 & 41.1 \\
$1 / 20$ & 18.8 & 27.1 \\
\hline
\end{tabular}

Scores calculated on basis of percent of time during each 15-sec trial in which movement is reported, expressed as a percent of $15 \mathrm{sec}$.

flashes more deliberate than they otherwise might have been.

An analysis of the size of the eye movements made in response to the light flashes indicates that eye movements varied in size between 10.5 and $15.6 \mathrm{deg}$, with the average eye movement covering about $13 \mathrm{deg}$ of visual angle. An eye movement of $13 \mathrm{deg} \mathrm{did}$, in fact, carry the eye from its focus at the center of the oscilloscope screen to either light, a distance of $62.5 \mathrm{~mm}$. Variations in the size of an eye movement did not appear to affect movement reports. Reports of movement were no more frequent or accurate when eye movements were on the large end of the eye-movement range than when they were on the small end of this range. Variations in the size of the eye movements did, of course, affect the absolute amount of target movement that accompanied any given eye movement. Since these variations in the size of eye movements did not seem to affect movement reports, the concommitant variations in the absolute amount of target movement also seem to have had no affect on these reports. The ability to perceive target movement seems therefore to depend on the $\mathrm{em} / \mathrm{tm}$ ratio rather than on the absolute amount of target movement. Table 4 describes the range of variation in the amount of target movement produced by variations in eye movements in the various $\mathrm{em} / \mathrm{tm}$ conditions.

\section{NDEM}

The results of this condition are not simply described. Only two quantitative descriptions of the data are possible; one is the time during each $15-\mathrm{sec}$ trial in which the $O$ reports seeing target motion, expressed as a percent of $15 \mathrm{sec}$. The other is the number of movement reports following eye movements of a certain extent, for example, larger than $3 \mathrm{deg}$, expressed as the percent of the total number of eye movements of that extent or larger occurring in any given 15-sec trial. Both ways of analyzing the data were used.

One of the drawbacks of a condition of spontaneous eye movements for an investigation of this kind is that $O s$ tend to make very few spontaneous eye movements that are larger than $1 \mathrm{deg}$ under these experimental conditions, i.e., when the only visual target is a dimly glowing spot of light which moves only as some function of the O's own eye movements and which they are instructed simply to observe. When the em/tm level is low, the amount of target displacement accompanying a relatively small eye movement is infinitesimal. Even with eye movements as large as $3 \mathrm{deg}$, the amount of target displacement is almost negligible. Thus there is only a slim chance that there are a sufficient number of accasions in which even a control $O$ is able to detect target movement. Furthermore, in this condition, Os are required to monitor the behavior of the target light constantly, which is likely to cause fatigue and consequent inaccuracies.

Table 5 depicts the results of the NDEM condition. Despite these drawbacks, the results obtained generally resemble those of the DEM condition. Comparing each $\mathrm{O}$ with himself as a control, the difference between the amount, the percent of each 15 sec in which movement was reported, for trials in which the em/tm ratio was $1 / 2$, $2 / 5,1 / 5,1 / 10$, and $1 / 20$ are as follows: $3.6 \%, 4.0 \%, 5 \%, 10 \%$, and $12 \%$, respectively. In the $1 / 2,2 / 5$, and $1 / 5$ conditions, the differences in amount of movement reported by both control and experimental Os are trivial, whereas the differences between experimental and control scores when the $\mathrm{em} / \mathrm{tm}$ was either $1 / 10$ or $1 / 20$ are significant. This same pattern appears if a comparison is made between the number of movement responses made by experimentals and controls following eye movements $3 \mathrm{deg}$ or larger at each of the em/tm levels (again treating each $\mathrm{O}$ as his own control). These differences are as follows for the $2 / 5,1 / 5$, and $1 / 10 \mathrm{em} / \mathrm{tm}$ trials: $11 \%, 11.4 \%$, and $17.7 \%$, respectively. The only significant difference is between experimental and control scores in the $1 / 10 \mathrm{em} / \mathrm{tm}$ condition.

Here, as in the DEM condition, direction of target displacement did not significantly affect movement reports. More important, in both conditions there is a significant difference between experimental and control $O$ s where the em/tm level is equal to $1 / 10$, and for both conditions this is the first level of $\mathrm{em} / \mathrm{tm}$ that produces a clear and significant difference between experimental and control Os. Also in both conditions displacement ratios less than $1 / 10$ invariably lead to a significant decrement on the part of experimental Os in the detection of target movement.

In the NDEM condition for both experimental and control Os a decrease in $\mathrm{em} / \mathrm{tm}$ level produced a corresponding decrease in the amount of movement reported or in the number of movement reports. This can be seen in the DEM results as well. A clear difference, however, between this data and the data from the DEM condition is the marked degree of similarity between the control and experimental scores. The decrease in the amount of movement reported by experimentals and controls with decreases in $\mathrm{em} / \mathrm{tm}$ level appears almost as striking in the control as in the experimental data. We attribute this similarity between experimental and control scores to the difficulty in detecting any target movement in this condition. This may be explained by the fact that the smaller the $\mathrm{em} / \mathrm{tm}$ ratio the less the actual target displacement, and the less the actual target displacement the less likely was the experimental $O$ to make eye movements that were sufficiently large to displace the target a detectable amount. It is therefore not surprising that both experimental and control Os show a decrease in ability to detect movement with a decrease in level of $\mathrm{em} / \mathrm{tm}$ that appears to be independent of whether or not target displacements occur during the $O$ 's eye movements.

Despite this similarity between the pattern of experimental and control scores, the pattern of significant differences is not totally unlike that found in the DEM data. As in the DEM results, the only significant difference that appears in the control scores is that between the scores obtained when the $\mathrm{em} / \mathrm{tm}$ equaled $1 / 20$ and the scores obtained from all other $\mathrm{em} / \mathrm{tm}$ levels. For experimental Os, the scores obtained from trials in which the em/tm ratio was $2 / 5$ or larger are significantly different from scores obtained on trials in which the em/tm was less than 2/5. This difference is not found in the DEM results in which the first significant break is between scores obtained from trials in which this ratio is less than $1 / 5$. NDEM results show no significant difference between scores from the $1 / 5$ and $1 / 10$ conditions. Thus, with spontaneous eye movements, target movement is no more difficult to perceive when that movement is dependent on the eye movement and $1 / 5$ as great as the eye movement than when it is $1 / 10$ as great. As in the DEM results, there is a significant difference between all experimental scores obtained when the 
Table 6

Mean Percent Correct Reports: DEM (Closer Viewing Distance)

\begin{tabular}{lccccc}
\hline & V+ & V- & H+ & H- & Grand $\bar{X}$ \\
\hline $2 / 5$ & 98.8 & 96.3 & 95.0 & 87.5 & 94.4 \\
$1 / 5$ & 92.5 & 86.1 & 71.9 & 58.9 & 77.2 \\
$1 / 10$ & 72.2 & 55.1 & 43.3 & 44.4 & 53.5 \\
$1 / 20$ & 35.7 & 33.5 & 7.5 & 20 & 24.1 \\
\hline
\end{tabular}

$\mathrm{em} / \mathrm{tm}$ ratio was $1 / 20$ and when the ratio was $1 / 10$ or greater.

That our results are not limited to eye movements of a particular absolute size is clear both from the results of the NDEM condition and from another study which predated the one here described. This earlier study resembled the DEM condition of the study reported with the following exceptions: The Os sat closer to the oscilloscope screen; the bite bar was placed $12.5 \mathrm{~cm}$ from the screen so that an eye movement of $26 \mathrm{deg}$ was required to move the eye from the center of the screen to one of the lights; and no control condition was employed. Each $\mathbf{O}$ was run for two experimental testing sessions but Os were not tested in pairs. In every other way, this experiment was identical to the DEM condition of the experiment reported. Ten Os were tested. The results appear in Table 6. With a few exceptions, they resemble those results already reported. The following are the exceptions: V $1 / 5$ scores are notably greater than the scores in the $\mathrm{H} 1 / 5$ conditions, although this difference is not significant at .05 . (The critical value for a difference that is significant at .05 is 35.165 , Scheffé, 1953. The difference value obtained is 23.3.) This trend, which was absent in the data previously described but present in all but the $2 / 5$ condition in this experiment, conforms to data reported by Wallach, Frey, and Rodney (1969) for head movement accompanied by abnormal displacements. Displacements that are at right angles to head movement appear to be more easily perceived. It is not clear why this tendency is restricted to this set of data. Another exception is that the mean scores in the $2 / 5$ condition are significantly greater than those in the $1 / 5$ condition. (Critical value for a difference at .05 is 16.3. The difference obtained is 17.2.) A difference between a displacement ratio of $2 / 5$ and one of $1 / 5$ does appear in the NDEM data. The means obtained with an $\mathrm{em} / \mathrm{tm}$ of $1 / 20$, with the exception of the $\mathrm{H}+1 / 20$, are greater than the analogous scores in the previously reported DEM results. This may reflect the fact that in the previously reported results when the $\mathrm{em} / \mathrm{tm}$ was $1 / 20$ an average eye movement displaced the target $.78 \mathrm{deg}$, whereas here the target was displaced $1.3 \mathrm{deg}$.

The point to be made is that despite some differences all results indicate that when target displacements are $2 / 5$ as great or greater than a concurrent eye movement (in the main experiment, when target displacements are 1/5 as great or greater), that displacement is predictably perceived, and when the displacement is only $1 / 20$ as great as the eye movement that displacement is generally not perceived.

\section{DISCUSSION}

When the eye scans a stationary object, the image of that object displaces across the retina of the eye in the same direction and to the same extent that the eye has moved. This is the normal condition for perceiving position constancy and is identical with the experimental condition in which the $\mathrm{em} / \mathrm{tm}$ level is zero and there is no objective target displacement. A look at the data obtained in the DEM condition where the $\mathrm{em} / \mathrm{tm}$ is zero indicates that, in fact, movement is generally not reported under these circumstances. Target displacements $1 / 5$ as great or greater than the accompanying eye movement were as reliably perceived as the identical target displacements that were independent of eye movements. This result conclusively establishes the fact that target displacements concurrent with eye movements are not suppressed and that there is no complete saccadic suppression of movement perception during saccadic eye movements. Results in the DEM conditions support the conclusion that direction of movement perceived corresponds to the actual direction of target displacement.

A second conclusion to be drawn is that these results support the concept of a comparator mechanism that matches available target displacement information with available information about $O$ eye movements.

If this hypothetical comparator were to operate with extreme precision, only a retinal-image displacement that exactly matched an eye movement would constitute the condition for perceived position constancy, that is, no movement perceived despite retinal-image movement. Thus, if the eye turned $13 \mathrm{deg}$ to the right, only a 13-deg rightward displacement over the retina would constitute a position-constancy match. It is apparent from the data that eye-movement/imagemovement comparisons are not so precise. In most instances when the target displacement is $1 / 5$ as large as the eye movement, regardless of the direction, the displacement is perceived. Thus, if, for example, the eye moves $13 \mathrm{deg}$ right and the target shifts $2.6 \mathrm{deg}$ in the same $(\mathrm{H}+)$ or the opposite $(\mathrm{H}-)$ direction, the image of the target will displace either 11.4 or
$15.6 \mathrm{deg}$ to the right over the retina, and movement will be seen. This seems to indicate that when the discrepancy between eye and image movement is $20 \%$ or greater, object movement will always be seen. If, however, the eye moves and the target displaces $1 / 20$ of that distance, movement is generally not seen. Thus, if the eye moves $13 \mathrm{deg}$ to the right and the target moves $.65 \mathrm{deg}$ either in the same $(\mathrm{H}+)$ or the opposite $\left(\mathrm{H}_{-}\right)$) direction, the image will displace either 12.35 or $13.65 \mathrm{deg}$ right over the retina. Under these circumstances, movement will not generally be seen, indicating that a discrepancy of $5 \%$ between eye and image displacement is acceptable and constitutes grounds for the perception of a stable, unmoving visual environment. That a 5\% target displacement is equivalent to target immobility can be seen by comparing the scores in the DEM condition when the $\mathrm{em} / \mathrm{tm}$ was $1 / 20$ with those scores obtained when the em/tm was zero. To make the two scores comparable, we can subtract the scores in the $1 / 20$ condition from $100 \%$, arriving at a mean score of no movement reports amounting to $88.2 \%$, which is entirely comparable to the score of 93.2 in the 0 condition in which there is actually no target displacement.

The fact that target displacements that are $1 / 10$ as large as concurrent eye movements are not perceived as frequently as the identical displacements occurring independently of eye movements, but are more frequently seen than displacements that are only $1 / 20$ as large as the eye movement, suggests that a discrepancy as great as $10 \%$ between eye and image displacement is close to the threshold of what constitutes an acceptable basis for perceiving stability during eye movements.

It might be argued that these results are simply to be interpreted as an index of the capacity for movement detection during saccadic eye movements. Looked at in this way the results indicate that the direction of target displacement during a saccade is not a factor in determining whether or not that displacement will be perceived, and, further, that there is the expected raising in the movement detection threshold during an eye movement. While this account of the results is legitimate, it in no way invalidates or contradicts an account of the result in terms of a comparator mechanism. To insist on an account of the data strictly in terms of thresholds and to rule out as illegitimate any other kind of account is to confuse description and explanation. The concept of a colisparator or compensatory mechanism is explanatory. The results of these experiments support this kind of explanation. To speak of threshold is simply to employ a well understood and 
efficient terminology for describing results. To infer from these results to the operation of a comparator mechanism is an attempt to account for them.

A puzzling aspect of the results is that they fail to show any consistent effect of the direction of target displacement. For the most part, the results indicate that the $\mathrm{em} / \mathrm{tm}$ is the central variable. Since under normal position-constancy conditions, the image of a stationary object displaces in the same direction over the retina as the eye moves, it might be reasonable to expect that experimental conditions in which the image of the target displaces in the same direction as the eye might lead less frequently to perceived movement, all other things being equal, than those cases in which the image displaces in some other direction. The only results that even suggest that this may be the case are from the early DEM study in which the $O$ sat rather close to the oscilloscope screen. As reported, the mean percent correct scores at each level of $\mathrm{em} / \mathrm{tm}$ are here somewhat higher for the two $\mathrm{V}$ conditions than for the two $\mathbf{H}$ conditions. This is exactly what one might expect, since in both $H$ conditions, regardless of the level of $\mathrm{em} / \mathrm{tm}$, the image of the target displaced in the same direction as the eye moved, whereas in the $V$ conditions the target was always displacing at right angles to the eye so that the image was always displacing at an angle to the direction the eye moved. No difference could be expected between the $H$ conditions, and generally none appears. Further testing is required in which image displacement is directly opposite to the direction of eye movement before any conclusions are drawn about the relevance of the direction of image displacement for the perception of target movement.

Another question left unanswered by this work is whether it is the percent of discrepancy between eye and image movement or the absolute discrepancy between these two inputs that determines if object movement will be seen. It is possible that whenever there is a discrepancy between eye and image movement that is larger than $10 \%$, target movement will generally be seen-or it is possible that whenever the discrepancy between eye and image movement is greater than, for example, $1.5 \mathrm{deg}$, movement will be seen. The fact that the results of the various conditions show the same pattern suggests that it is the percent of discrepancy which is crucial, but more work is required.

One could ask why the postulated comparator operation during eye movements is not more precise. This question is unresolved by this study, but several possibilities suggest themselves. Perhaps the fact that a $5 \%$ mismatch between eye and visual displacement information is an acceptable basis for position constancy during eye movements is a function of a saccadic inhibition of visual displacement detection during eye movements such that a 10-deg displacement is indistinguishable from a 9-deg displacement.

Still another possibility might be that the tolerance for a mismatch as a basis for position constancy may be the result of a faulty memory for egocentric direction. If the crucial components in the comparison between eye and visual displacement information were eye-movement information on the one hand and information about the visual direction of an object before and after an eye movement on the other hand, rather than information about retinal movement, then the tolerance for a mismatch might be the result of an inherent inability to remember visual directions accurately. If an object were straight ahead at the onset of an eye movement and moved $1 \mathrm{deg}$ during an eye movement of $10 \mathrm{deg}$, perhaps the reason that target movement is not perceived is the result of an inability to remember precisely where the object was at the onset of the eye movement. If this were the case, a 1 -deg displacement might be indistinguishable from a 0-deg displacement and would account for the fact that it is not perceived. In considering this explanation it is necessary to assume not only that memory for position becomes more inaccurate when the position to be remembered precedes an eye movement but, more important, that retinal movement is still registered in the match between information about eye movement and change of visual direction, since otherwise we would have to conclude that no real movement is perceived during an eye movement. If retinal movement information did not play a role, only the inference of movement based on a change in visual direction would be possible. From our own work, it would be extremely hard to argue that real movement is not seen during a saccade, since Os reported no difference in the appearance of target movement when they were tested under experimental and control conditions.

Finally, it might be argued that the apparent imprecision in the match between eye and target movement is a function of inaccurate or imprecise eye-movement information. If the eyes were to turn $10 \mathrm{deg}$ to look at something off to the side, and the information registered in the nervous system is that the eye has moved either more or less than $10 \mathrm{deg}$, perhaps somewhere between 9 and $10 \mathrm{deg}$, then a 1-deg image displacement in either the same or the opposite direction as the eye moved would "match" the eye movement, and no movement should be perceived. The results of this experiment do not permit any evaluation of the relative merits of these possible explanations of the apparent imprecision in the "match" between eye and image movement.

These results reconfirm the fact that retinal displacement cannot be considered a sufficient condition for movement perception since in all cases in which target displacements are in the same direction as the eye movement $(\mathrm{H}+)$, the higher the $\mathrm{em} / \mathrm{tm}$ level the less the retinal image displacement, yet the lower the $\mathrm{em} / \mathrm{tm}$ level the less likely is target movement to be perceived. In conclusion, these results clearly contradict those reported by Wallach and Lewis (1965) and extend those of Mack and Bachant (1969). They constitute evidence that "abnormal" retinal movements concurrent with eye movements do lead to perceived movement.

\section{REFERENCES}

BRINDLEY, G. S., \& MERTON, P. A. The absence of position sense in the human eye. Journal of Physiology, 1960, 153, 127-130.

HAY, J. Visual adaptation to an altered correlation between eye movements and head movements. Science, 1968, 160, 429-430.

MACK, A., \& BACHANT, J. Perceived movement of the afterimage during eye movements Perception \& Psychophysics, 1969, 6 379.384

MacKAY, D. M. Ways of looking at perception. In W. Wathen-Dunn (Ed.), Models for the preception of speech and visual form. Cambridge: M.L.T. Press, 1967.

ROCK, I. The nature of perceptual adaptation New York: Basic Books, 1966.

SHAFFER, O., \& WALLACH, H. Extent-of-motion thresholds under subject-relative and object-relative conditions. Perception \& Psychophysics, 1966, 1, 447-451.

TEUBER, H, L. Perception. In J. Field (Ed.), Handbook of physiology-neurophysiology III. Baltimore: Williams \& Wilkins, 1960. Pp. 1595-1668.

von HOLST, E. Relations between the central nervous system and the peripheral organs. British Journal of Animal Behaviour, 1954, 2, 89-94.

WALLACH, H., \& FREY, K. Adaptation in the constancy of visual direction measured by a one-trial method. Perception \& Psychophy sics, $1970,5,249-252$

WALLACH, H., FREY, K., \& RODNEY, G. Adaptation to field displacement during head movement unrelated to the constancy of visual direction. Perception \& Psychophysics, 1969, S, 253-256.

WALLACH, H., \& KRAVITZ, J. The measurement of the constancy of visual direction and of its adaptation. Psychonomic Science, 1965, 2, 217-218. 


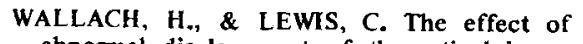
abnormal displacement of the retinal image during eye movements. Perception \& Psychophysics, 1966, 1, 25-29.

WINER, B. J. Statistical principles in experimental design. New York: MoGraw-Hill, 1962.

YARBUS, A. L. Eye movements and vision. New York: Plenum Press, 1967.

\section{NOTES}

1. This research was done during an NIMH postdoctoral fellowship at Stanford University. I would like to express my appreciation to everyone who helped make this research possible. I am particularly grateful to Dr. Leo Ganz for his generous help and to Robert Brown who designed and built some of the apparatus and assisted in the testing of Os. I would also like to express my gratitude to Hans Wallach. Irvin Rock, and Leon Festinger for their careful reading of an earlier version of this manuscript. 2. Address: New School for Social Research. New York, New York 10003.

3. All discussion of movement perception is restricted to object movement, which is subject relative since, with movement that is object relative, the relative displacement of one object in the visual tietd with respect to another is a powerful indicator of movement.

4. We assume that the information about eye movements that is matched against retinal displacement information is efferent rather than afferent information (Brindley \& Merton, 1960).

5. Data reported by Schaffer and Wallach (1966) indicates that Os are capable of reporting far smaller subject-relative target displacements. Perhaps the low intensity of the target in our work accounts for this difference in results.

(Accepted for publication January 28, 1970.) 